\title{
Motion of a Test Particle in the Kerr-Newman De/Anti De Sitter Space-Time
}

\author{
Shanjit Heisnam¹, Irom Ablu Meitei ${ }^{2}$, Kangujam Yugindro Singh1 \\ ${ }^{1}$ Department of Physics, Manipur University, Canchipur, Imphal, India \\ ${ }^{2}$ Department of Physics, Pettigrew College, Ukhrul, India \\ Email: shanjit@iucaa.ernet.in, ablu.irom@gmail.com, yugindro361@gmail.com
}

Received 8 April 2014; revised 6 May 2014; accepted 14 May 2014

Copyright (C) 2014 by authors and Scientific Research Publishing Inc.

This work is licensed under the Creative Commons Attribution International License (CC BY).

http://creativecommons.org/licenses/by/4.0/

(c) $($ i) 0 pen Access

\begin{abstract}
In this paper we obtain the geodesic equations of motion of a test particle (charged particle and photon) in the Kerr-Newman de/anti de Sitter black hole by using the Hamilton-Jacobi equation. We determine the positions of the inner, outer and cosmological horizons of the black hole. In terms of the effective potentials, the trajectory of the test particle within the inner horizon is studied. It appears that there are stable circular orbits of a charged particle and photon within the inner horizon and that the combined effect of the charge and rotation of the Kerr-Newman de/anti de Sitter black hole and the coupling between the charge of the test particle and the electromagnetic field of the black hole may account for this.
\end{abstract}

\section{Keywords}

Geodesic Equations, Kerr-Newman De/Anti De Sitter Black Hole, Inner and Outer Event Horizons, Stable Circular Orbits

\section{Introduction}

The solutions of Einstein-Maxwell equations in the Kerr-Newman de/anti de Sitter space-time in the presence of the cosmological constant, $\Lambda$, give rise to the geometrical structure of a Kerr-Newman black hole and its singularity. The geometrical structure is asymptotically de sitter at large times when a repulsive cosmological constant, $\Lambda>0$, is considered and may contain a cosmological horizon with a dynamic background and for an attractive cosmological constant. $\Lambda<0$, the geometrical structure, is asymptotically anti de Sitter and contains black hole horizons. The recent serious study of the observational and theoretical aspects of a small positive cosmological constant term which may be relevant at the present epoch [1] [2] and the accurate measurements of the anisotropy of the cosmic relic background radiation and the observational analysis of type $\mathrm{I}_{\mathrm{a}}$ supernova with high red 
shift parameter $Z \leq 1$ in the framework of the inflationary cosmology [3]-[6], suggests that a repulsive cosmological constant $\Lambda>0$ has to be taken seriously for understanding the properties of the presently observed universe. On the other hand, it is recognized that the de/anti de Sitter space-time has an important role in the multidimensional string theory [7].

According to Chandrasekhar [8], there exist two general types of particle orbits in the black hole gravitational field: orbits of the first kind, which are completely confined outside the black hole event horizon, and orbits of the second kind, which penetrate inside the black hole. In the case of rotating or charged black hole, there are bound periodic planetary orbits, known as the orbits of the third kind, which neither come out nor terminate at the central singularity [9]. The third kind of bound orbits inside the black hole horizon were discussed by [10] [11] for charged particle in the vicinity of the rotating charged black holes and by [12] for neutral particle.

The geometrical properties of the Kerr-Newman de/anti de sitter space-time with non zero cosmological constant are described by the geodesic equations of motion of a test particle. The motion of a test charged particle in the gravitational field of a charged black hole is fully described by three integrals of motion namely, $E$, the total particle energy, $L_{\varphi}$, the azimuthal component of the angular momentum and $Q$, the Carter constant [13].

In Section 2, we review general geodesic orbits of test particle in the Kerr Newman de/anti de sitter spacetime. In Section 3, we discuss the bound stable periodic orbits for a charged particle and photon inside the inner horizon. Section 4 is a brief conclusion. We use the units $\mathrm{G}=\mathrm{c}=1$ throughout the paper.

\section{General Geodesic Orbits in the Kerr-Newman De/Anti De Sitter Space-Time}

The equations of motion of a test particle of mass $\mathrm{m}$ and charge $\varepsilon$ in the gravitational field of a rotating charged black hole in the Kerr-Newman de/anti Sitter space-time may be determined from the principle of least action with the action defined as [14],

$$
S=\int_{1}^{2}\left(-m^{2}+\varepsilon A_{i} \dot{x}^{i}\right) \mathrm{d} \lambda
$$

where $\mathrm{d} \lambda=\frac{1}{m} \mathrm{~d} \tau, \mathrm{d} \tau=\sqrt{-g_{i j} \mathrm{~d} x^{i} \mathrm{~d} x^{j}}$ being the proper time of the test charged particle along geodesics;

$A_{i}=(-\phi, \mathbf{A})$ is the covariant four vector potential and a dot overhead a symbol denotes differentiation with respect to the parameter $\lambda$. From the action, the Lagrangian is identified as

$$
L=\frac{1}{2} g_{i j} \dot{x}^{i} \dot{x}^{j}+\varepsilon A_{i} \dot{x}^{i}
$$

with the normalizing condition,

$$
g_{i j} \dot{x}^{i} \dot{x}^{j}=-m^{2} .
$$

Effecting the variation of the action, one obtains

$$
\delta S=\left.\left(g_{i j} \dot{x}^{j}+\varepsilon A_{i}\right) \delta x^{i}\right|_{1} ^{2}+\int_{1}^{2}\left[-m \frac{\mathrm{d} u_{i}}{\mathrm{~d} \tau}+\varepsilon F_{i j} u^{j}\right] \delta x^{i} \mathrm{~d} \tau
$$

where $F_{i j}=\frac{\partial A_{j}}{\partial x^{i}}-\frac{\partial A_{i}}{\partial x^{j}}$ is the electromagnetic field tensor and $u^{j}=\frac{\mathrm{d} x^{j}}{\mathrm{~d} \tau}$. The integrated term vanishes at both limits as the end points are fixed. According to the principle of least action, $\delta S=0$ for the correct path of motion of the particle. This condition leads to the following equations of motion for the test charged particle in the given field:

$$
\frac{\mathrm{d}^{2} x^{i}}{\mathrm{~d} \tau^{2}}=\frac{\varepsilon}{m} F_{i j} \frac{\mathrm{d} x^{j}}{\mathrm{~d} \tau}
$$

The Hamiltonian corresponding to the Lagrangian (2) is found as

$$
H=\frac{1}{2} g^{i j}\left(p_{i}-\varepsilon A_{i}\right)\left(p_{j}-\varepsilon A_{j}\right)
$$

where 


$$
p_{i}=g_{i j} \dot{x}^{j}+\varepsilon A_{i}
$$

are the canonical momenta. Since $H$ does not depend explicitly on $\lambda$, the Hamiltonian is a constant of motion. As such, by using the normalization condition, one finds

$$
H=-\frac{1}{2} m^{2} .
$$

In the standard Boyer-Lindquist coordinates $(t, r, \theta, \varphi)$, the metric describing the Kerr-Newman de/anti de Sitter space-time takes the form,

$$
\begin{aligned}
\mathrm{d} s^{2}= & -\frac{\Delta_{r}}{\rho^{2}}\left[\frac{\mathrm{d} t}{I}-\frac{a \sin ^{2} \theta \mathrm{d} \phi}{I}\right]^{2} \\
& +\frac{\Delta_{\theta} \sin ^{2} \theta}{I^{2}}\left[\frac{a \mathrm{~d} t}{\rho}-\frac{\left(r^{2}+a^{2}\right) \mathrm{d} \phi}{\rho}\right]^{2} \\
& +\rho^{2}\left(\frac{\mathrm{d} r^{2}}{\Delta_{r}}+\frac{\mathrm{d} \theta^{2}}{\Delta_{\theta}}\right)
\end{aligned}
$$

where the functions $\rho, \Delta_{r}, \Delta_{\theta}$ and $I$ are defined respectively by

$$
\begin{aligned}
& \rho^{2}=r^{2}+a^{2} \cos ^{2} \theta \\
& \Delta_{r}=\left(1-\frac{1}{3} \Lambda r^{2}\right)\left(r^{2}+a^{2}\right)-2 M r+e^{2} \\
& \Delta_{\theta}=\left(1+\frac{1}{3} \Lambda a^{2} \cos ^{2} \theta\right) \\
& I=1+\frac{1}{3} \Lambda a^{2} .
\end{aligned}
$$

On the other hand, the electromagnetic field for the source is given by the required vector potential:

$$
A_{\mu}=\frac{e r}{I \rho^{2}}\left[\delta_{\mu}^{t}-a \sin ^{2} \theta \delta_{\mu}^{\phi}\right]
$$

The corresponding nonzero contravariant components $g^{i j}$ of the metric are obtained as

$$
\begin{aligned}
& g^{t t}=\frac{I^{2}}{\Delta_{r} \Delta_{\theta} \rho^{2}}\left[\Delta_{r} a^{2} \sin ^{2} \theta-\Delta_{\theta}\left(r^{2}+a^{2}\right)^{2}\right] \\
& g^{\phi t}=g^{t \phi}=\frac{I^{2}}{\Delta_{r} \Delta_{\theta} \rho^{2}}\left[\Delta_{r}-\Delta_{\theta}\left(r^{2}+a^{2}\right)^{2}\right] \\
& g^{\phi \phi}=I^{2}\left[\frac{1}{\sin ^{2} \theta \Delta_{\theta} \rho^{2}}-\frac{a^{2}}{\Delta_{r} \rho^{2}}\right] \\
& g^{r r}=\frac{\Delta_{r}}{\rho^{2}} \\
& g^{\theta \theta}=\frac{\Delta_{\theta}}{\rho^{2}} .
\end{aligned}
$$

The geometrical properties of the metric element given by Equation (9) can be analyzed by taking into account the limiting conditions: $\Lambda \rightarrow 0, M \rightarrow 0, e \rightarrow 0$ in the metric. By imposing these limiting conditions, one finds $\mathrm{d} s^{2}=-\mathrm{d} t^{2}+\rho^{2}\left(\frac{\mathrm{d} r^{2}}{r^{2}+a^{2}}+\mathrm{d} \theta^{2}\right)+\left(r^{2}+a^{2}\right) \sin \theta \mathrm{d} \phi^{2}$ which is actually the flat space-time in the spheroidal coordinates under the coordinate transformation: $x=\sqrt{r^{2}+a^{2}} \sin \theta \cos \phi ; \quad y=\sqrt{r^{2}+a^{2}} \sin \theta \sin \phi$; 
$z=r \cos \theta$ in the ranges of $r \geq 0,0 \leq \theta \leq \pi, 0 \leq \phi \leq 2 \pi$ leading to the standard Cartesian form. In the $\theta=\pi / 2$ plane, $r=0$ represents a ring disk of radius $a$ with properties different from the usual radial coordinate. If we consider the limit $a \rightarrow 0$, the metric recovers the Reissner-Nordstrom de/anti de Sitter metric which, in standard spherical coordinates, is expressed as:

$$
\mathrm{d} s^{2}=-\Delta_{r} \mathrm{~d} t^{2}+\frac{\mathrm{d} r^{2}}{\Delta_{r}}+r^{2}\left(\mathrm{~d} \theta^{2}+\sin ^{2} \theta \mathrm{d} \phi^{2}\right)
$$

where $\Delta_{r}=1-\frac{\Lambda r^{2}}{3}-\frac{2 M}{r}+\frac{e^{2}}{r^{2}}$. Under the limiting condition. $M \rightarrow 0, e \rightarrow 0, a \rightarrow 0$, we see the de/anti de sitter space-time with the flipping of the sign of $\Lambda$. When $a \rightarrow 0, e \rightarrow 0$ we recover the Schwarzchild de/anti Sitter space-time.

Since the metric is stationary and axisymmetric, it is clear that there exists two Killing vector fields given by $\xi_{(t)} \equiv\left(\frac{\partial}{\partial t}\right)_{r, \theta, \phi} ; \xi_{(\phi)} \equiv\left(\frac{\partial}{\partial \phi}\right)_{t, r, \theta}$. We find two constants of motion namely, the energy and angular momentum of the test particle corresponding to the dot product of the four-momentum with the Killing vector: $\mathrm{P} \cdot \xi_{(t)}=-E$ and $\mathrm{P} \cdot \xi_{(\varphi)}=L$. By (6), the general form of the Hamilton-Jacobi equation is

$$
\frac{\partial S}{\partial \lambda}=-\frac{m^{2}}{2}=\frac{1}{2} g^{i j}\left[\frac{\partial S}{\partial x^{i}}-\varepsilon A_{i}\right]\left[\frac{\partial S}{\partial x^{j}}-\varepsilon A_{j}\right]
$$

whose solution takes the form,

$$
\begin{gathered}
S=-\frac{1}{2} m^{2} \lambda-E t+L \phi+\int \frac{r}{\Delta_{r}} \mathrm{~d} r+\int \frac{\theta \sqrt{\mathrm{V}(\theta)}}{\Delta_{\theta}} \mathrm{d} \theta \\
\text { where } R(r)=I^{2}\left[E\left(r^{2}+a^{2}\right)-a L+\frac{e r \varepsilon}{I}\right]^{2}-\Delta_{r}\left(Q+I^{2}(a E-L)^{2}+m^{2} r^{2}\right)
\end{gathered}
$$

and

$$
V(\theta)=\left(Q+I^{2}(a E-L)^{2}-m^{2} a^{2} \cos ^{2} \theta\right) \Delta_{\theta}-\frac{I^{2}}{\sin ^{2} \theta}\left(a E \sin ^{2} \theta-L\right)^{2} .
$$

Here $\mathrm{Q}$ is the Carter constant. Using the action given in Equation (14), the following differential equations governing the motion of the test particle can be deduced:

$$
\begin{gathered}
\rho^{2} \frac{\partial r}{\partial \lambda}= \pm \sqrt{R(r)} \\
\rho^{2} \frac{\partial \theta}{\partial \lambda}= \pm \sqrt{V(\theta)} \\
\rho^{2} \frac{\partial t}{\partial \lambda}=I^{2}\left(\mathrm{r}^{2}+a^{2}\right)\left[E\left(r^{2}+a^{2}\right)-a L+\frac{e r \varepsilon}{I}\right]-\frac{I^{2} a}{\Delta_{\theta}}\left(\mathrm{a} E \sin ^{2} \theta-\mathrm{L}\right) \\
\rho^{2} \frac{\partial \phi}{\partial \lambda}=-\frac{I^{2}}{\Delta_{\theta} \sin ^{2} \theta}\left(a E \sin ^{2} \theta-L\right)+\frac{I^{2} a}{\Delta_{r}}\left[E\left(r^{2}+a^{2}\right)-a L+\frac{e r \varepsilon}{I}\right] .
\end{gathered}
$$

where

$$
\begin{gathered}
\rho^{2}=r^{2}+a^{2} \cos ^{2} \theta \\
\Delta_{r}=\left(1-\frac{1}{3} \Lambda r^{2}\right)\left(r^{2}+a^{2}\right)-2 M r+e^{2} \\
\Delta_{\theta}=\left(1+\frac{1}{3} \Lambda a^{2} \cos ^{2} \theta\right)
\end{gathered}
$$




$$
I=1+\frac{1}{3} \Lambda a^{2}
$$

The functions $\mathrm{R}(\mathrm{r})$ and $\mathrm{V}(\theta)$ serve as the effective potentials defining the motion of the test particle in $r$ - and $\theta$-directions [15]. Thus, the study of a test particle in the gravitational field of the Kerr-Newman de/anti de Sitter space-time is reduced to the study of motion of the test particle in the effective potentials $R(r)$ and $\mathrm{V}(\theta)$. For a circular orbit for which $\dot{r}=0$, the following conditions are satisfied at some radius $r$ :

$$
\begin{gathered}
R(r)=0, \\
R^{\prime}(r) \equiv \frac{\mathrm{d} R(r)}{\mathrm{d} r}=0 .
\end{gathered}
$$

It may also be verified that the condition $R^{\prime \prime}(r)<0$ is satisfied showing that the circular orbit is stable.

\subsection{Discussion on Singularities and Event Horizons}

The Kerr-Newman de/anti de Sitter metric (9) have singularities at $\rho=0$ and at $\Delta_{r}=0$. The physically reasonable singularity is located at $\rho=0$ which is satisfied with $\theta=\pi / 2$ and $\mathrm{r}=0$. The condition $\Delta_{r}=0$ implies that the Kerr-Newman de/anti de Sitter metric (9) exhibits four radial horizons. These radial horizons may be found as the roots of the equation $\Delta_{r}=0$ which may be put in the form,

$$
-\frac{\Lambda}{3} r^{4}+\left(1-\frac{a^{2} \Lambda}{3}\right) r^{2}-2 M r+a^{2}+e^{2}=0
$$

The four roots are:

$$
\begin{aligned}
& r_{ \pm}=\frac{\sqrt{X}}{2} \pm \frac{1}{2}\left[A-B+\frac{(2 Z)^{\frac{1}{3}}}{3 L\left(Y+\sqrt{\left(Y^{2}-4 Z\right)}\right)^{\frac{1}{3}}}+\frac{\left(Y+\sqrt{\left(Y^{2}-4 Z\right)}\right)^{\frac{1}{3}}}{3 L 2^{\frac{1}{3}}}-\frac{4 M}{L \sqrt{X}}\right]^{\frac{1}{2}} \\
& r_{c}=-\frac{\sqrt{X}}{2}-\frac{1}{2}\left[A-B+\frac{(2 Z)^{\frac{1}{3}}}{3 L\left(Y+\sqrt{\left(Y^{2}-4 Z\right)}\right)^{\frac{1}{3}}}+\frac{\left(Y+\sqrt{\left(Y^{2}-4 Z\right)}\right)^{\frac{1}{3}}}{3 L 2^{\frac{1}{3}}}+\frac{4 M}{L \sqrt{X}}\right]^{\frac{1}{2}} \\
& r_{n}=-\frac{\sqrt{X}}{2}+\frac{1}{2}\left[A-B+\frac{(2 Z)^{\frac{1}{3}}}{3 L\left(Y+\sqrt{\left(Y^{2}-4 Z\right)}\right)^{\frac{1}{3}}}+\frac{\left(Y+\sqrt{\left(Y^{2}-4 Z\right)}\right)^{\frac{1}{3}}}{3 L 2^{\frac{1}{3}}}+\frac{4 M}{L \sqrt{X}}\right]^{\frac{1}{2}}
\end{aligned}
$$

in which

$$
\begin{gathered}
L=\frac{\Lambda}{3}, \quad A=\frac{\left(1-a^{2} L\right)}{3 L}, \quad B=\frac{\left(-1+a^{2} L\right)}{L}, Z=\left(1-14 a^{2} L-12 e^{2} L+a^{4} L^{2}\right)^{3}, \\
Y=72 L\left(a^{2}+e^{2}\right)\left(1-a^{2} L\right)+2\left(1-a^{2} L\right)^{3}-108 L M^{2} \\
X=-A-B-\frac{(2 Z)^{\frac{1}{3}}}{3 L\left(Y+\sqrt{\left(Y^{2}-4 Z\right)}\right)^{\frac{1}{3}}}-\frac{\left(Y+\sqrt{\left(Y^{2}-4 Z\right)}\right)^{\frac{1}{3}}}{3 L 2^{\frac{1}{3}}} .
\end{gathered}
$$


Three out of the four roots of Equation (27), have physical interpretations as follows: $r_{+}$and $r_{-}$are the outer and inner event horizons of the black hole; $r_{c}$ is the cosmological horizon for an observer between $r_{+}$ and $r_{c}$. Using the L. Ferrari's method [16], it may be shown that the real solutions for the horizon Equation (27) are controlled by a factor $h$, called the horizon parameter, defined as

$$
\begin{aligned}
h \equiv & \frac{1}{16 \Lambda^{3}}\left[4\left(a^{2}+e^{2}\right)-\frac{1}{\Lambda}\left(1-\frac{\Lambda a^{2}}{3}\right)^{2}\right]^{3} \\
& +\frac{1}{16 \Lambda^{4}}\left[\left(1-\frac{\Lambda a^{2}}{3}\right)\left\{\frac{1}{\Lambda}\left(1-\frac{\Lambda a^{2}}{3}\right)^{2}+12\left(a^{2}+e^{2}\right)\right\}-18 M^{2}\right]^{2} .
\end{aligned}
$$

For the negative cosmological constant $\Lambda<0$, some particular cases arise:

1) $h>0$ : two real solutions, $r_{+}$and $r_{-}$are expected;

2) $h<0$ : no real solution exists, thus providing a naked singularity;

3) $h=0: r_{+}$and $r_{-}$coincide forming a single event horizon.

For the positive cosmological constant $\Lambda>0$, depending on the value of $h$, there arise several physical interpretations:

1) $h<0$ : the roots $r_{-}, r_{+}$and $r_{c}$ are all real and positive showing that the black hole has well-defined horizons.

2) $h=0$ : the event horizons, $r_{-}$and $r_{+}$become degenerate.

3) $h>0$, there exists only one horizon.

\subsection{Geometrical Surfaces of Kerr-Newman De/Anti De Sitter Space-Time}

In the Boyer-Lindquist coordinates, the stationary limit surfaces (SLS) of Kerr-Newman de/anti de Sitter black hole are obtainable by setting the roots $g_{t t} \equiv\left[\frac{\Delta_{\theta} a^{2} \sin ^{2} \theta-\Delta_{r}}{I^{2} \rho^{2}}\right]=0$. In the light of Equation (10), these conditions give rise to a fourth order equation,

$$
-\frac{\Lambda}{3} r^{4}+r^{2}\left(1-\frac{3 a^{2}}{\Lambda}\right)-2 M r+\left(1+\frac{a^{2} \Lambda \cos ^{2} \theta}{3}\right) a^{2} \sin ^{2} \theta+\left(a^{2}+e^{2}\right)=0 .
$$

By taking $C=\left(1+L a^{2} \cos ^{2} \theta\right) a^{2} \sin ^{2} \theta+a^{2}+e^{2}$, the four roots of (32) are found as

$$
\begin{aligned}
& r_{S E / I}=\frac{\sqrt{X}}{2} \pm \frac{1}{2}\left[A-B+\frac{(2 Z)^{\frac{1}{3}}}{3 L\left(Y+\sqrt{\left(Y^{2}-4 Z\right)}\right)^{\frac{1}{3}}}+\frac{\left(Y+\sqrt{\left(Y^{2}-4 Z\right)}\right)^{\frac{1}{3}}}{3 L 2^{\frac{1}{3}}}-\frac{4 M}{L \sqrt{X}}\right]^{\frac{1}{2}} \\
& r_{S C}=-\frac{\sqrt{X}}{2}-\frac{1}{2}\left[A-B+\frac{(2 Z)^{\frac{1}{3}}}{3 L\left(Y+\sqrt{\left(Y^{2}-4 Z\right)}\right)^{\frac{1}{3}}}+\frac{\left(Y+\sqrt{\left(Y^{2}-4 Z\right)}\right)^{\frac{1}{3}}}{3 L 2^{\frac{1}{3}}}+\frac{4 M}{L \sqrt{X}}\right]^{\frac{1}{2}} \\
& r_{S}=-\frac{\sqrt{X}}{2}+\frac{1}{2}\left[A-B+\frac{(2 Z)^{\frac{1}{3}}}{3 L\left(Y+\sqrt{\left(Y^{2}-4 Z\right)}\right)^{\frac{1}{3}}}+\frac{\left(Y+\sqrt{\left(Y^{2}-4 Z\right)}\right)^{\frac{1}{3}}}{3 L 2^{\frac{1}{3}}}+\frac{4 M}{L \sqrt{X}}\right]^{\frac{1}{2}}
\end{aligned}
$$

in which 


$$
\begin{gathered}
L=\frac{\Lambda}{3}, \quad A=\frac{\left(1-a^{2} L\right)}{3 L}, B=\frac{\left(-1+a^{2} L\right)}{L}, Z=\left(1-2 a^{2} L-12 C L+a^{4} L^{2}\right)^{3}, \\
Y=72 L C\left(1-a^{2} L\right)+2\left(1-a^{2} L\right)^{3}-108 L M^{2}, \\
X=-A-B-\frac{(2 Z)^{\frac{1}{3}}}{3 L\left(Y+\sqrt{\left(Y^{2}-4 Z\right)}\right)^{\frac{1}{3}}}-\frac{\left(Y+\sqrt{\left(Y^{2}-4 Z\right)}\right)^{\frac{1}{3}}}{3 L 2^{\frac{1}{3}}} .
\end{gathered}
$$

For each radial horizon defined by (27) there is an associated stationary limit surface (SLS) defined by (32). Both the hyper surfaces given by Equations (27) and (32) coincide at $\theta=0, \pi$. The conditions for the existence of two distinct interior and exterior horizons for $\Lambda<0$ and three real horizons, interior, exterior and cosmological horizons for $\Lambda>0$ roots are respectively given by $X_{\text {erg }}>0$ (for $\Lambda<0$ ) and $X_{\text {erg }}<0$ (for $\Lambda>0$ ) where

$$
\begin{aligned}
X_{\text {erg }} \equiv & \frac{1}{16 \Lambda^{3}}\left[4\left(a^{2}+e^{2}\right)-\frac{1}{\Lambda}\left(1-\frac{\Lambda a^{2}}{3}\right)^{2}-4 a^{2} \sin ^{2} \theta\left(1+\frac{\Lambda a^{2} \cos ^{2} \theta}{3}\right)\right]^{3} \\
& +\frac{1}{16 \Lambda^{4}}\left[\left(1-\frac{\Lambda a^{2}}{3}\right)\left\{\frac{1}{\Lambda}\left(1-\frac{\Lambda a^{2}}{3}\right)^{2}+12\left(a^{2}+e^{2}\right)-12 a^{2} \sin ^{2} \theta\left(1+\frac{\Lambda a^{2} \cos ^{2} \theta}{3}\right)\right\}-18 M^{2}\right]^{2} .
\end{aligned}
$$

\subsection{Circular Orbit of a Test Charged Particle inside the Inner Horizon}

We discuss the non rotating charged black hole: $a=0, m=1, Q=0$. With the help of Equations (25) and (26), we obtain a pair of coupled equations for the energy $E$ and angular momentum $L$ of the test charged particle:

$$
\left(E r^{2}+e r \varepsilon\right)^{2}-\left(r^{2}-\frac{1}{3} \Lambda r^{4}-2 r+e^{2}\right)\left(L^{2}+r^{2}\right)=0
$$

and

$$
\begin{aligned}
& 2\left(E r^{2}+e r \varepsilon\right)(2 E r+e \varepsilon)-\left(L^{2}+r^{2}\right)\left(-2+2 r-\frac{4 r^{3} \Lambda}{3}\right) \\
& -2 r\left(-2 r+r^{2}+e^{2}-\frac{\Lambda r^{4}}{3}\right)=0 .
\end{aligned}
$$

As the roots of the Equations (37) and (38) we obtain the following the pairs of values for $E$ and $L$ :

$$
\begin{gathered}
E_{1,2}= \pm \frac{\Delta D_{1}-e \varepsilon\left(-4 r+r^{2}+3 e^{2}+\frac{\Lambda r^{4}}{3}\right)}{2 r\left(-3 r+r^{2}+2 e^{2}\right)} \\
L_{1,2}^{2}=\frac{r^{2}}{\left(r^{2}-3 r+2 e^{2}\right)}\left[\left(r-e^{2}-\frac{\Lambda r^{4}}{3}\right)+\frac{e \varepsilon \Delta\left(e \varepsilon \pm D_{1}\right)}{2\left(r^{2}-3 r+2 e^{2}\right)}\right]
\end{gathered}
$$

where

$$
\Delta=e^{2}-2 r+r^{2}-\frac{\Lambda r^{4}}{3}
$$

and $D_{1}^{2}=\left(8 e^{2}+e^{2} \varepsilon^{2}-12 r+4 r^{2}\right)$. The stability condition $R^{\prime \prime}(r)<0$ for the non rotating circular orbit in the region of $0<r<r_{-}$is satisfied for $\left(E_{1}, L_{1}\right)$ for all $\varepsilon>m$. The interaction between the charge of the particle and the electromagnetic field of the black hole may account for the existence of stable circular orbit of the 
charged particle inside the inner horizon [7].

\subsection{Photon Orbit inside the Inner Horizon}

The photon orbit is obtained in the case of the ultra relativistic limit for very massive particle energy $E \rightarrow \infty$. This limiting situation is equivalent to the situation when $m=0$. Such photon orbits mainly depend on two parameters: the azimuthal impact parameter $b=L / E$ and the latitudinal impact parameter given by $q=Q / E^{2}$. For photon orbit, we have

$$
\varepsilon=0, m=0, I=1+\frac{\Lambda a^{2}}{3} \Delta_{r}=\left(1-\frac{1}{3} \Lambda r^{2}\right)\left(r^{2}+a^{2}\right)-2 M r+e^{2}
$$

From (25) and (26), we find:

$$
\begin{aligned}
R(r)= & I^{2}\left[E\left(r^{2}+a^{2}\right)-a L\right]^{2} \\
& -\left[\left(1-\frac{1}{3} \Lambda r^{2}\right)\left(r^{2}+a^{2}\right)-2 r+e^{2}\right]\left[Q+I^{2}(a E-L)^{2}\right]=0
\end{aligned}
$$

and

$$
\begin{aligned}
R^{\prime}(r) & =I^{2}\left[E\left(r^{2}+a^{2}\right)-a L\right] 2 E r \\
& -\left(-1-\frac{2}{3} \Lambda r^{3}-\frac{\Lambda r a^{2}}{3}+r\right)\left[Q+I^{2}(a E-L)^{2}\right]=0 .
\end{aligned}
$$

On solving for $b=L / E$, we get the following expression as roots of (41) and (42):

$$
\begin{gathered}
b_{1}=\frac{1}{a\left(1+\frac{2}{3} \Lambda r^{3}+\frac{\Lambda r a^{2}}{3}-r\right)}\left[r\left(r-3 r+2 e^{2}\right)+a^{2}\left(1+r+\frac{\Lambda r a^{2}}{3}\right)\right] \\
b_{2}=\frac{\left(r^{2}+a^{2}\right)}{a}
\end{gathered}
$$

Using these values of $b_{1}$ and $b_{2}$ given by (43) and (44) back into (41) and (42) we get a pair of values for $q$ :

$$
\begin{gathered}
q_{1}=\frac{I^{2} r^{2}}{a^{2}\left(1-r+\frac{2}{3} \Lambda r^{2}+\frac{\Lambda a^{2} r}{3}\right)^{2}} \\
{\left[4 a^{2}\left(\frac{a^{2} r^{2} \Lambda}{6}-\frac{a^{2} r^{4} \Lambda^{2}}{9}+r-e^{2}-r^{3} \Lambda+\frac{2}{3} r^{2} e^{2} \Lambda\right)-\left(-3 r+r^{2}+2 e^{2}\right)^{2}\right]} \\
q_{2}=\frac{-I^{2} r^{4}}{a^{2}}
\end{gathered}
$$

The condition for stability $R^{\prime \prime}(r)<0$ is satisfied for $\left(b_{1}, q_{2}\right)$ in the region of $0<r<r_{-}$. The stable circular orbit may exist within the inner horizon which appears to be the result of combined effects of both rotation and charge of the black hole. Such an interesting feature was observed initially by Balek et al. 1989 [7].

\section{Conclusion}

We have shown that charged particles and photons may have stable periodic orbits inside the inner horizon of Kerr-Newman de/anti de Sitter black hole. The interaction between the charge of the particle and the electromagnetic field of the black hole may account for existence of stable circular orbits in the case of a charged particle inside the inner horizon. However, in the case of a photon inside the inner horizon, its stable circular orbit may be due to the combined effect of the rotation and the charge of the black hole. 


\section{Acknowledgements}

The authors would like to thank the Inter-University Centre for Astronomy and Astrophysics (IUCAA), Pune for providing hospitality and support during the preparation of the paper. Shanjit Heisnam is grateful to the University Grants Commission for providing him financial assistance in the form of UGC-BSR fellowship.

\section{References}

[1] Sahni, V. and Starobinsky, A. (2000) The Case for a Positive Cosmological $\Lambda$-Term. International Journal of Modern Physics D, 9, 373-443.

[2] Sahni, V. (1990) Energy Density of Relic Gravity Waves from Inflation. Physical Review D, 42, 453-463. http://dx.doi.org/10.1103/PhysRevD.42.453

[3] Perlmutter, S., et al. (1998) Discovery of a Supernova Explosion at Half the Age of the Universe. Nature, 391, 51-54.

[4] Perlmutter, S., et al. (1997) Measurements of the Cosmological Parameters $\Omega$ and $\Lambda$ from the First Seven Supernovae at $\mathrm{z} \geq 0.35$. Astrophysical Journal, 483, 565-581.

[5] Riess, A.G., et al. (1998) Observational Evidence from Supernova for an Accelerating Universe and a Cosmological Constant. Astronomical Journal, 116, 1009-1038.

[6] Perlmutter, S. (1999) Measurement of $\Omega$ and $\Lambda$ from 42 High-Redshift Supernovae. Astrophysical Journal, 517, 565586. http://dx.doi.org/10.1086/307221

[7] Sen, A. (1998) Developments in Superstring Theory.

[8] Chandrasekhar, S. (1983) The Mathematical Theory of Black Holes. Clarendon Press, Oxford.

[9] Dokuchaev, V.I. (2010) Is There Any Life inside Black Hole? Classical and Quantum Gravity, 28, 235015-235026.

[10] Bicak, J., Stuchlik, Z. and Balek, V. (1989) The Motion of Charged Particles in the Field of Rotating Charged Black Holes and Naked Singularities-I. Bulletin of the Astronomical Institutes of Czechoslovakia, 40, 65-92.

[11] Balek, V., Bicak, J. and Stuchlik, Z. (1989) The Motion of the Charged Particles in the Field of Rotating Charged Black Holes and Naked Singularities-II. Bulletin of the Astronomical Institutes of Czechoslovakia, 40, 133-165.

[12] Hackmann, E., Kagramanova, V., Kunz, J. and Lammerzahl, C. (2010) Analytical Solution of the Geodesics Equation in Kerr-(anti)de Sitter Space-Times. Physical Review D, 81, 044020-38. http://dx.doi.org/10.1103/PhysRevD.81.044020

[13] Carter, B. (1968) Global Structure of the Kerr Family of Gravitational Fields. Physical Review, 174, 1559-1571. http://dx.doi.org/10.1103/PhysRev.174.1559

[14] Padmanabhan, T. (2010) Gravitation: Foundations and Frontier. Cambridge University Press, Cambridge. http://dx.doi.org/10.1017/CBO9780511807787

[15] Bardeen, J.M., Press, W.H. and Teukolsky, S.A. (1972) Rotating Black Holes: Locally Nonrotating Frames, Energy Extraction and Scalar Synchrotron Radiation. Astrophysical Journal, 178, 347-369. http://dx.doi.org/10.1086/151796

[16] Gerolamo, C. (1545) Ars Magna or the Rules of Algebra. Dover. 